\title{
Inclusión educativa del adulto mayor en la UAEM
}

\section{Educational inclusion in the elderly in the UAEM}

\author{
MORENO-AGUIRRE, Alma Janeth $\dagger$, ORTIZ-RODRÍGUEZ, María Araceli"*, CRUZ-ABARCA, \\ Laura y CAPISTRÁN-PÉREZ, Luz Patricia
}

'Facultad de Comunicación Humana;"Facultad de Nutrición, Universidad Autónoma del Estado de Morelos (UAEM). Avenida Universidad 1001, Chamilpa, 62209 Cuernavaca, Mor.

ID $1^{\mathrm{er}}$ Autor: Alma Janeth, Moreno-Aguirre / ORC ID: 0000-0001-9076-5987, CVU CONACYT ID: 173543

ID $1^{\text {er }}$ Coautor: María Araceli, Ortiz-Rodríguez / ORC ID: 0000-0003-0847-0261, CVU CONACYT ID: 449164

ID $2^{\text {do }}$ Coautor: Laura, Cruz-Abarca / ORC ID: 0000-0001-8580-0768, CVU CONACYT ID: 478996

ID $3^{\text {er }}$ Coautor: Luz Patricia, Capistrán-Pérez / ORC ID: 0000-0002-4261-3521, CVU CONACYT ID: 774201

DOI: $10.35429 / J E T .2019 .9 .3 .1 .9$

Recibido: 05 de Julio, 2019; Aceptado 28 de Septiembre, 2019

\section{Resumen}

Objetivo. Analizar las necesidades que presentan y los apoyos que se requieren para el proceso de inclusión educativa del adulto mayor en educación superior en la UAEM. Metodología. Se realizó un análisis de investigaciones previas en tema de condiciones, necesidades y apoyos a considerar para lograr la inclusión educativa en población de estudiantes adultos mayores, además de conocer el proceso y seguimiento que se brinda dentro de la UAEM para cumplir este objetivo. Los apoyos otorgados dentro la institución son fundamentales, así como considerar los factores propios del estudiante adulto mayor como son: el cognitivo, el neurobiológico con o sin presencia de enfermedad crónica degenerativa entre otras, además del psicológico donde la parte socioafectiva es muy importante. Contribución. Los resultados analizados permitirán fortalecer la inclusión educativa en población de estudiantes con estas características a nivel superior y ofrecer nuevas estrategias de apoyo.

\begin{abstract}
Objective. Analyze the needs they present and the support required for the educational inclusion process of the elderly in higher education in the UAEM. Methodology. An analysis of previous research was carried out in terms of conditions, needs and supports to be considered to achieve educational inclusion in the population of older students, as well as knowing the process and monitoring that is provided within the UAEM to meet this objective. The supports granted within the institution are fundamental, as well as considering the factors of the older adult student such as: cognitive, neurobiological with or without the presence of chronic degenerative disease among others, in addition to the psychological one where the socio-affective part is very important.Contribution. The analyzed results will strengthen the educational inclusion in the student population with these characteristics at a higher level and offer new support strategies.
\end{abstract}

Senior adult, Educational inclusion, Cognitive impairment
Adulto mayor, Inclusión educativa, Deterioro
cognitivo 9: 1-9.

\footnotetext{
* Correspondencia del Autor (correo electrónico: araceli.ortiz@uaem.mx)

$\dagger$ Investigador contribuyendo como primer autor.
} 


\section{Introducción}

En México, la persona considerada como Adulto Mayor de acuerdo al H. Congreso de la Unión, es aquella que tiene sesenta años de edad o más y que se encuentra domiciliada o en tránsito en territorio nacional (Saldaña-Ibarra \& Hernández-Guerson, 2017). La Organización Mundial de la Salud (OMS), define al adulto mayor sano como aquel con condición autónoma, aunque tenga deficiencia en su salud, mantiene una capacidad funcional, definiendo la funcionalidad como la capacidad de poder desarrollar sus actividades sociales, de orden cognitivo y educativas en el cumplimiento de sus actividades instrumentales de la vida diaria. Otras investigaciones manejan el concepto de "fragilidad" en el adulto mayor, refiriendo que es la disminución del rendimiento de los múltiples sistemas fisiológicos, que conducen al final a un agotamiento de la reserva funcional del adulto mayor con riesgos de discapacidad y dependencia (Mogollón, 2012).

La población mexicana, tiene una tendencia en la pirámide poblacional con un mayor rango de individuos en edad avanzada, según reportes estadísticos del Consejo Nacional de Población (CONAPO) de cada 10 mexicanos tres son menores de 15 años y sólo uno tiene 60 años, sin embargo para 2050 se prevé que sea la misma cantidad de jóvenes y de adultos mayores, aproximadamente $20.7 \%$ de menores de 15 años y $21.5 \%$ de adultos mayores, lo que implica modificar políticas de salud, sociales, laborales y educativas (CONAPO, 2012).

Teniendo este factor como un curso natural del proceso biológico, por el impacto que el mismo tiene en varias áreas, se pretende resaltar el reto que se va a enfrentar en el país con un alto índice de población en edad avanzada, que en la mayoría de los casos es probable que presente deterioro cognitivo (Benavidez-Caro, 2017; Camargo-Hernández \& Laguado, 2017).

El deterioro cognitivo se define como una falta de habilidad en funciones cognitivas como memoria, atención y fluidez verbal, y funciones ejecutivas, por lo que es un reto que se debe enfrentar y ocuparse en forma eficiente de recomendaciones que contribuyan al bienestar de esta población (Benavidez-Caro, 2017; Carrascal \& Solera, 2014; Monteagudo-Torres et al., 2009).
$\mathrm{Si}$ a futuro se tiene contemplado el incremento de la población adulta mayor, con probabilidad de riesgo que pueda presentar deterioro cognitivo, es fundamental proyectar programas que tiendan a promover el desarrollo cognitivo (Camargo-Hernández \& LaguadoJaimes, 2017; Monteagudo-Torres et.al., 2009).

Desde esta perspectiva es necesario enfatizar la intervención en programas a nivel primario y a otros niveles y contextos como el educativo, el laboral y el social (Ramírez \& Víctor-Ramírez, 2010; Sánchez-Gil \& Pérez, 2008). Una estrategia para ello, es dar oportunidades de aprendizaje a la persona adulta mayor que permitan interactuar para aumentar el saber y desarrollo de competencias para la vida cotidiana, lograr una adaptación y al mismo tiempo ser incluidos en su entorno. Estos aprendizajes permiten no solo el conocer los cambios propios del envejecimiento sino que al analizar toda esta experiencia también se obtiene información importante para identificar con mayor precisión las necesidades actuales y promover nuevas propuestas (Saldaña-Ibarra \& Hernández-Guerson, 2017).

La OMS ha apoyado e implementando políticas de manejo de la demencia y las alteraciones cognitivas, en población del adulto mayor y en otras poblaciones. En algunos países como en Cuba, existen políticas enfocadas en el manejo de adultos mayores, y pacientes con riesgo de demencia, como programas de actividad física para el adulto mayor, programas de control de enfermedades crónicodegenerativas, programas para evitar el tabaquismo, etc. (Benavidez-Caro, 2017).

Estos programas apoyan los derechos humanos del adulto mayor y han generado un aumento de la esperanza de vida y con ello nuevas necesidades en la persona adulta mayor. Una de estas necesidades es continuar su formación académica, que al mismo tiempo impactará en el fortalecimiento y mantenimiento del estado cognitivo, así como su estado neurobiológico y socio-afectivo en general, permitiendo una mejor calidad de vida $\mathrm{e}$ inclusión en los diferentes escenarios de sus actividades cotidianas. 
Existen varias iniciativas en diferentes países de instituciones públicas y privadas de educación superior, en formar unidades académicas para fomentar la formación en educación superior en el adulto mayor tales como Chile, Perú, Nicaragua, Costa Rica, Argentina, Brasil, República Dominicana, Cuba, Uruguay y México (Monteagudo-Torres et al., 2009; Saldaña-Ibarra \& Hernández-Guerson, 2017). Con estos antecedentes, se plantea que la persona ubicada como adulto mayor, tiene la capacidad de seguir cumpliendo un rol importante en la producción de bienes y servicios, ya que la mitad de la población entre los 60 a 64 años de edad en México, continúa como población económicamente activa y otro porcentaje menor con edades mayores. Muchos de estos casos las personas presentan condiciones socio-económicas y educativas en rezago. Por ello es importante rescatar a esta población activa con programas no solo de salud sino de oferta educativa que les pueda abrir mejores expectativas, ya que la esperanza de vida cada vez es mayor (Narro-Robles, Martuscelli- Quintana, \& Barzana-García, 2012; Ramírez \& Víctor-Ramírez, 2010). Por lo tanto, el propósito de esta investigación es analizar en el adulto mayor las necesidades en los aspectos: cognitivo, neurobiológico y socio-afectivo, así como apoyos requeridos para el proceso de inclusión educativa a nivel superior y con ello fortalecer los procesos actuales que se están llevando a cabo en la Universidad Autónoma de Estado de Morelos (UAEM).

\section{Método}

Mediante una revisión de documentos en relación al tema de inclusión educativa en el adulto mayor, se utilizaron varias fuentes; en mayor cantidad información obtenida en línea (red), verificando su confiabilidad y veracidad. Se realizó una búsqueda durante el periodo mayo - agosto de 2019, se agruparon varios documentos los cuales oscilaron alrededor de 43 , determinados por las palabras clave como son deterioro cognitivo, vejez, adultos mayores, funcionamiento cognitivo e inclusión educativa. Se identificaron varias fuentes seleccionado las que eran más específicas al tema a investigar $(\mathrm{N}=15)$ y sobre ellas se realizó el análisis de las condiciones y necesidades que deben tomarse en cuenta en población de estudiantes que son adultos mayores y con ello establecer propuestas para lograr la inclusión educativa.
Se identificó por información reportada de las estrategias de apoyo que son expuestas por otras instituciones a nivel internacional y nacional, así también las llevadas a cabo en la UAEM a través de diferentes instancias, como el Programa Universitario para la Inclusión Educativa y Atención a la Diversidad en nivel superior en población de estudiantes adultos mayores (UAEM, 2012-2018b).

Los apoyos otorgados dentro la institución son fundamentales, sin olvidar también de gran impacto la intervención o manejo de los factores propios del estudiante adulto mayor como son: el cognitivo, el neurobiológico con o sin presencia de enfermedad crónica degenerativa y el aspecto socio-afectivo.

\section{Resultados}

La información obtenida en la búsqueda y análisis se organizó de la siguiente manera que a continuación se presenta:

Antecedentes históricos de la educación superior para el adulto mayor.

En el siglo pasado en el año de 1973, en Toulouse, Francia, Pierre Vellas propone el proyecto de la formación de las Universidades de la Tercera edad (UTE). Esta propuesta tuvo como objetivo dar a las personas adultas mayores un programa de actividades que respondiera a sus condiciones, necesidades y aspiraciones en esa etapa de la vida, integrándose a este proyecto la formación de la Asociación Internacional de Universidades de la Tercera Edad (AIUTA) (Saldaña-Ibarra \& Hernández-Guerson, 2017).

Posterior a la fundación de la primera UTE y con apoyo de la AIUTA, se promovió la formación de nuevas UTE en países europeos y en los EE.UU. Diversos autores plantearon que de acuerdo a sus características de organización y propósitos las clasificaron en tres grupos: 1) El Modelo Francés, 2) El Modelo Británico o de Cambridge y 3) El Modelo Híbrido o Mixto (Ramírez \& Víctor-Ramírez, 2010; SaldañaIbarra \& Hernández-Guerson, 2017). 
Durante poco más de cuarenta décadas la AIUTA reúne a universidades de todo el mundo, con el propósito de formar a los adultos mayores a través de la educación permanente, los intercambios universitarios y la investigación científica (Saldaña-Ibarra \& HernándezGuerson, 2017; Valle-Aparicio, 2014).

Se han identificado diversas razones por las que se considera que las UTE fueron creadas, una de ellas es que mejora de las condiciones de vida ya que evita el aislamiento socio-cultural y humano, impacto en el aspecto psico-biológico que fomenta un estado cognitivo saludable que le permite al adulto mayor no solo realizar actividades cotidianas sino nuevas perspectivas de vida en el ámbito académico, laboral, social, entre otros. Con ello se plantean también cambios a las políticas públicas, así como la educación favorece un campo de crecimiento del grupo de adultos mayores como nuevo mercado para los sectores empresariales (Saldaña-Ibarra \& Hernández-Guerson, 2017).

Los países en América Latina no se han quedado atrás, uno de los pioneros fue la Pontificia Universidad Católica de Chile, que en el año de 1988, creó el Programa Adulto Mayor, en base a las necesidades de mejorar la calidad de vida por el acelerado incremento de su población adulta mayor. Las líneas de acción que generó este programa fueron la docencia, la investigación, los proyectos sociales, las publicaciones y las políticas públicas (SaldañaIbarra \& Hernández-Guerson, 2017).

Posteriormente, en año de 2006, el trabajo realizado por la Pontificia Universidad Católica de Chile a través del Programa para el Adulto Mayor, junto con la AIUTA, el gobierno Finlandés, el Banco Interamericano de Desarrollo (BID) y la colaboración de universidades de Nicaragua, Costa Rica y Perú desarrollaron el proyecto "Apoyo a las Universidades de la Tercera Edad y Entrenamiento Profesional", donde se propusieron dos líneas de acción: 1) La capacitación y entrenamiento profesional mediante diplomados a distancia y 2) $\mathrm{La}$ difusión, divulgación e intercambio de experiencias universitarias.
El trabajo de esta alianza promovió la creación de la Red de Universidades de la Tercera Edad (RED UTE), uniéndose a esta red Argentina, Brasil, Chile, Cuba, República Dominicana, Uruguay y México (Saldaña-Ibarra \& Hernández-Guerson, 2017; Velis, Viteri, Terranova \& Ordoñez, 2018).

La educación superior para el adulto mayor en México.

Con la participación de México en la RED UTE, logró fundar la primera Universidad de la Tercera Edad en el país en la ciudad de México en el año 2009. Para el año 2012 ya se contaban con dos planteles teniendo como líneas de acción: desarrollo humano, social, artístico, tecnológico, cultural, salud, activación física, del cuidado del medio ambiente, idiomas, historia de México, filosofía medieval, entre otros (Saldaña-Ibarra \& Hernández-Guerson, 2017: Velis et al., 2018).

En el año 2011, la Universidad Nacional Autónoma de México (UNAM), creó el Seminario Universitario Interdisciplinario sobre Envejecimiento y Vejez (SUIEV), teniendo como objetivo la construcción de redes, actividades de colaboración y proyectos interdisciplinarios, que promuevan a la UNAM. Para el 2013 a través del SUIEV, se formó la Red Universitaria de Envejecimiento y Vejez, conformada por 21 entidades académicas, con el objetivo de profundizar en el análisis y estudio del adulto mayor desde distintos enfoques en investigación, formación, actualización y capacitación para los adultos mayores (SaldañaIbarra \& Hernández-Guerson, 2017; NarroRobles, Martuscelli- Quintana \& BarzanaGarcía, 2012).

En el 2010, la Facultad de Estudios Superiores Zaragoza a través de la Unidad de Investigación en Gerontología, inició el Curso Universitario de Envejecimiento Activo para Adultos Mayores (CUEAAM), con el objetivo de proporcionar formación universitaria a personas adultas mayores de la Ciudad de México y promover su desarrollo integral durante la etapa de la vejez, a partir de brindar herramientas para plantearse futuros con mejor calidad de vida (Saldaña-Ibarra \& HernándezGuerson, 2017). 
Otros estados se unieron a esta propuesta como lo fueron Nuevo León, Puebla, Yucatán y Oaxaca. La Universidad Autónoma de Nuevo León (UANL), en 2011, con la Universidad para los Mayores propuso el objetivo de brindar nuevas oportunidades de crecimiento personal, académico y desarrollo sustentable a las personas de 55 años y más a través de diplomados, cursos y talleres. En ese mismo año se creó la Universidad Binacional de la Tercera Edad (U3E), dirigida por la Universidad Regiomontana, A.C. (UR), el Centro de Servicios Integrales para el Adulto Mayor A.C. (CESIAM) y la Asociación de Asilos y Casas de Retiro del Estado de Nuevo León A.C. (ACARE), con el objetivo de integrar a los Adultos Mayores a la sociedad, contribuir en el derecho una vida con calidad; recibir educación y capacitación para el trabajo (Saldaña-Ibarra \& Hernández-Guerson, 2017; Vázquez-Galicia, 2009).

En Puebla, la
Iberoamericana propuso universidad
programa universitario que tuvo como objetivo fortalecer el conocimiento y la experiencia que los adultos mayores a través de un diplomado en el cual se abordaron diversos contenidos como: el mundo contemporáneo, la apreciación de las artes, las ciencias sociales, el diálogo fe y cultura y el desarrollo humano (Saldaña-Ibarra \& Hernández-Guerson, 2017).

En el 2014 en la ciudad de Mérida, Yucatán; la Universidad Marista, brindó un espacio a los adultos mayores para desarrollar sus potencialidades humanas, adquirir herramientas para una vida en plenitud y enriquecer sus conocimientos culturales en un ambiente fraterno, a través de un diplomado donde se abordan algunos de los temas como: Alimentación saludable; Crecimiento interior; Cine y sociedad, Desarrollo humano, Gimnasia cerebral, entre otros (Saldaña-Ibarra \& Hernández-Guerson, 2017).

Otra acción es la que se brindó en el estado de Oaxaca con la Universidad del Adulto Mayor (UNIDAM), a través del Diplomado del Adulto Mayor Anfitrión de Oaxaca, donde se capacita a adultos mayores como anfitriones, para atender las necesidades de otras personas mayores que visitan Oaxaca.
Este diplomado tiene un contexto en el ámbito del turismo y considera dos áreas formativas:

Desarrollo Humano y Gestión del Turismo, donde se trabajan contenidos como: Derecho; El poder de las palabras; Autoestima; Al encuentro de mí mismo; El placer de mantenerse joven; Salud y Nutrición; Creatividad en el Arte; Cuentos con Ciencia; Historias de Vida entre otros (Saldaña-Ibarra \& Hernández-Guerson, 2017).

Perspectivas actuales en México de las UTE.

En el 2002, la Organización de las Naciones Unidas (ONU), planteó en su informe de la Segunda Asamblea Mundial sobre el Envejecimiento, que el adulto mayor tiene derecho a su independencia, a la alimentación, vivienda, educación, y también a la protección jurídica, que lo salvaguarde de la exclusión mencionado en el artículo 12, en la página 4:

"Las personas de edad deben tener la oportunidad de trabajar hasta que quieran y sean capaces de hacerlo en el desempeño de trabajos satisfactorios, productivos y de seguir teniendo acceso a la educación y a los programas de capacitación” (Mogollón, 2012, p 57).

Todos los antecedentes que se mencionan muestran evidencias que indican que se ha avanzado en la creación de un marco normativo y de políticas públicas que responden a los acuerdos internacionales a favor de los derechos y necesidades de las personas adultas mayores; por lo que en la actualidad aún quedan muchos retos a resolver en el ámbito nacional como en el internacional.

Uno de estos retos fue la propuesta de construir la Universidad Veracruzana del Adulto Mayor bajo un eje de un programa intergeneracional, con la participación de sus académicos, quienes también muchos de ellos se encuentran como población adulta mayor. 
Sin embargo, no solo en la Universidad Veracruzana se observa población académica de adultos mayores; en otras instituciones de educación superior tal es el caso de la UAEM, con la formación el 22 de marzo del 2013 en acuerdo unánime del Consejo Universitario en instrumentar el Programa Universitario de Inclusión Educativa y Atención a la Diversidad que en su informe del 2015-2017, reportó en su Segundo Estudio de Prevalencia de la Diversidad en la UAEM a 320 adultos mayores (89 mujeres y 231 hombres), clasificados en las siguientes categorías: 2 estudiantes, 269 académicos y 49 trabajadores administrativos, que para la fecha actual la cifra de estas categorías se ha incrementado (Saldaña-Ibarra \& Hernández-Guerson, 2017; Universidad Autónoma del estado de Morelos [UAEM], s.f.).

Consideraciones, necesidades y apoyos del adulto mayor para lograr incluirse en el contexto educativo.

El adulto mayor debido a los procesos propios del envejecimiento puede entrar en situación de vulnerabilidad, esta situación puede ser: la físico-emocional, la del contexto social (teorías de la desvinculación, la de la actividad y la de la continuidad), la del aspecto cognitivo (declinación, tanto en la memoria, el lenguaje, abstracción y en las funciones ejecutivas), entre otras (Mogollón, 2012; Sánchez-Gil \& Pérez, 2008).

Otro estudio reportó de manera más detallada las categorías más importantes de la declinación cognitiva como: razonamiento inductivo, velocidad de percepción, habilidad numérica, habilidad verbal, memoria verbal y orientación espacial (Hedden \& Gabrieli, 2004; citado en Mogollón, 2012). Además se ha demostrado evidencia científica que el envejecimiento (adulto mayor) muestra un deterioro físico debido a que se asocia cambios músculo-esqueléticos con el decremento de la capacidad máxima de consumo de oxígeno comparado con una persona adulta joven; que condiciona además pérdida de masa muscular en personas mayores a 70 años en un promedio de 1\% al año (Mogollón, 2012). Estos cambios en los diferentes contextos que sufre el adulto mayor, abren una perspectiva de ofrecer expectativas para ofrecer mejor calidad de vida, sin olvidar la potencialidad que presentan en desarrollar nuevos aprendizajes en esta etapa de la vida.
Se plantea en recientes investigaciones la plasticidad cerebral en el adulto mayor, que es también significativa incluso en poblaciones mayores a 80 años de edad, por lo que hay un incremento de las competencias educativas debido al amplio rango de actividades intelectuales que pueda tener. Por lo tanto la plasticidad cerebral favorece el desarrollo de reserva cognitiva, interpretando este aspecto que el adulto mayor fomenta la capacidad de mantener las funciones ejecutivas superiores activas (lóbulo frontal) incrementando el aprendizaje y la educación.

De acuerdo a estudios clínicos se consideran dos evidencias de que existe una hipótesis acerca de la reserva cognitiva, una de estas evidencias es un estudio que analizó como repercute el aspecto educativo y laboral en la tasa metabólica cerebral local para la glucosa rCMRglc de 242 pacientes con enfermedad de Alzheimer, 72 con deterioro cognitivo amnésico leve y su grupo control de 144 participantes saludables. Se encontró en el estudio de tomografía de emisión de positrones, una fuerte correlación entre altos niveles de educación y ocupación contra un bajo metabolismo de la glucosa en la corteza temporoparietal posterior y en el precuneus de los pacientes con alguna de las patologías ya mencionadas, con ello se demuestra que la educación y actividad laboral pueden ser potenciadores de la reserva cognitiva del cerebro, aminorando el nivel clínico histopatológico de la enfermedad.

La segunda evidencia que apoya a esta teoría es el estudio a un grupo de 37 personas con demencia tipo Alzheimer y 161 sin demencia. Se les inyectó un agente radiactivo denominado Compuesto Pittsburgh B, marcado con Carbono 11 ([11C]-PIB), que se adhiere a las placas de amiloide relacionadas con la enfermedad de Alzheimer. Se les realizó una Tomografía por Emisión de Positrones, teniendo en cuenta que el [11C-PIB] permite la detección de esta proteína Beta-amiloide en el cerebro. Los resultados identificaron una correlación positiva en adultos mayores con niveles altos en educación y un mayor rendimiento cognitivo. 
Este estudio evidenció la presencia de placas cerebrales relacionadas con la enfermedad de Alzheimer, por lo que estos resultados le dan sustento empírico a la hipótesis planteada de que la reserva cognitiva influye en la asociación entre la carga patológica de la enfermedad de Alzheimer y la cognición, mejorando o manteniendo niveles de cognición aceptables para la educación del adulto mayor, esto direcciona que para que haya una buena reserva cognitiva y plasticidad neuronal, debe mantenerse una actividad neuronal permanente y esto se logra con una constante educación (Roe, et al.; 2007 citado en Mogollón, 2012).

De ahí que los resultados de manera empírica se fueron observando desde los años setenta de los beneficios que traía no solo en el aspecto social y afectivo sino también neurobiológico la apertura de las UTE.

Una de las estrategias actuales con un sustento sólido es continuar con la formación de programas que fomenten la educación superior en el adulto mayor, fundamentados en los beneficios que traen en los aspectos físico y emocional (Mogollón, 2012; Ramírez \& VíctorRamírez, 2010).

Otra estrategia es la capacitación a docentes para brindar una calidad educativa en una sociedad inclusiva, esto significa con una visión intercultural.

Esta capacitación debe ser dirigida y fortalecida hacia aspectos de justicia social, enseñanza inclusive y valor pedagógico a la diversidad de estudiantes debido a sus condiciones neurobiológicas, cognitivas, culturales y socio afectivas. El papel del docente es crucial para fomentar la identidad, el conocimiento y la comunicación (Nygård, P., Svensson, P., \& Karlsson, A., 2019).

Los apoyos que se identificaron para la población universitaria adulta mayor de la UAEM aún no son suficientes, pero se encuentran en desarrollo través del Programa Universitario de Inclusión Educativa y Atención a la Diversidad. Estos apoyos son:
Un Plan de acción donde se han eliminado barreras arquitectónicas, para asegurarse el ingreso y permanencia en las instalaciones universitarias, adecuaciones de acceso a la información en bibliotecas y centros de información documental, eliminar barreras administrativas para el ingreso a la universidad como la aplicación de un examen general diferenciado, adecuaciones curriculares a los programas de estudio, las Unidades de Servicios de Apoyo para la Inclusión Educativa (USAIEUAEM) y las Unidades Universitarias de Atención Comunitaria (UUAC). Lo que ahora se plantea es no solo abordar a los estudiantes de nivel superior sino a los trabajadores académicos y administrativos que también se encuentran dentro de este grupo de edad. El desarrollo de programas o estrategias internas a la institución permitirá no solo los beneficios de que se está generando inclusión educativa sino un impacto más allá fuera de las aulas que permite al adulto mayor una mejor calidad de vida (UAEM, 20122018 a,b).

\section{Agradecimiento}

Agradecemos al Cuerpo Académico Estudios Transdisciplinarios Sobre Grupos Vulnerables (UAEMOR-CA-142), a la Facultad de Comunicación Humana, Facultad de Nutrición y al Programa de Inclusión Educativa y Atención a la Diversidad de la UAEM por el apoyo brindado.

\section{Conclusiones y Recomendaciones}

El desarrollo y fortalecimiento de políticas educativas que favorezcan la inclusión en el adulto mayor es una tarea primordial, gracias a la atención a los factores de impacto que impiden esta inclusión como los ambientales, los socioculturales, los institucionales, los neurobiológicos propios del envejecimiento o los adquiridos por algunas patologías, así como el incremento de la esperanza de vida en la población mundial. La UAEM va en camino, obteniendo ya sus primeros frutos del trabajo realizado.

Es posible que la sociedad actual aún no se encuentre lista para enfrentar los cambios tanto a nivel educativo, laboral, económico, social o político, de la nueva visión de cómo se está conformando la población adulta mayor. 
Gracias a los avances en la investigación gerontológica, en las neurociencias y en las tecnologías de neuroimágen muestran hallazgos valiosos y otra perspectiva de la población adulta mayor, eliminando la visión de ser una población vulnerable ante los contextos de educación y el desarrollo social.

\section{Referencias}

Benavides-Caro, C.A. (2017). Deterioro cognitivo en el adulto mayor. Revista Mexicana de Anestesiología.40 (29), 107-112.

Camargo-Hernández, K. del C., LaguadoJaimes, E. (2017). Grado de deterioro cognitivo de los adultos mayores institucionalizados en dos hogares para ancianos del área metropolitana de Bucaramanga - Santander, Colombia. Rev Univ. Salud. 19(2), 163-170. DOI: http://dx.doi.org/10.22267/rus.171902.79

Carrascal, S. \& Solera, E. (2014). Creatividad y desarrollo cognitivo en adultos mayores. Arte, Individuo y Sociedad. 26 (1), 9-19. ISSN: 11315598

http://dx.doi.org/10.5209/rev_ARIS.2014.v26.n 1.40100

https://revistas.ucm.es/index.php/ARIS/article/d ownload/40100/41533/0

CONAPO (Consejo nacional de Población) (2012) Proyecciones de la población 2010-2050, CONAPO, México.

Mogollón, E. (2012). Una perspectiva integral del adulto mayor en el contexto de la educación. Revista Interamericana de Educación de Adultos, 34 (1), 56-74. ISSN: 0188-8838. http://www.redalyc.org/articulo.oa?id=4575450 90005

Monteagudo-Torres, M., Gómez-Viera, N., Martín-Labrador, M., Jiménez-Fontao, L., Mc Cook, E. \& Ruiz-García, D. (2009). Evaluación del estado cognitivo de los adultos mayores de 60 años, en un área de salud del Policlínico Docente Plaza de la Revolución. Revista Cubana de Medicina, 48(3), 59-70. http://scielo.sld.cu/scielo.php?script=sci_arttext \& pid=S0034-

75232009000300006\&lng=es\&tlng=es.
Narro-Robles, J., Martuscelli- Quintana, J. \& Barzana-García, E. (Coord.).(2012) Plan de diez años para desarrollar el Sistema Educativo Nacional. [En línea]. México: Dirección General de Publicaciones y Fomento Editorial, UNAM http://www.planeducativonacional.unam.mx

Nygård Larsson, P., Svensson Källberg, P., \& Karlsson, A. (2019). Educational inclusion and social cohesion: the diversity of contemporary pedagogy. In Understanding International Migration in the 21st Century: Conceptual and Methodological Approaches. 16th Annual IMISCOE Conference.

Ramírez, L. V. \& Víctor-Ramírez, A. C. (2010). Educación para adultos en el siglo XXI: análisis del modelo de educación para la vida y el trabajo en México ¿avances o retrocesos? Tiempo de Educar, 11(21), 59-78. ISSN: 1665-0824. http://www.redalyc.org/articulo.oa?id=3111616 3004

Sánchez-Gil, I. Y. \& Pérez, V. T. (2008). El funcionamiento cognitivo en la vejez: atención y percepción en el adulto mayor. El funcionamiento cognitivo en la vejez: atención y percepción en el adulto mayor. Revista Cubana de Medicina General Integral, 24(2) $\mathrm{http}: / /$ scielo.sld.cu/scielo.php?script=sci_arttext $\&$ pid=S08621252008000200011

Saldaña-Ibarra S.A. \& Hernández-Guerson E. (2017). Universidad del Adulto Mayor: Un nuevo escenario de desafíos y de oportunidades. Memoria de congreso electrónica: XIV Congreso Internacional de Investigación Educativa COMIE, San Luis Potosí 2017. Universidad Veracruzana. http://www.comie.org.mx/congreso/memoriaele ctronica/v14/doc/2599.pdf

UAEM. (2012-2018a). Manual Azul. http://www.uaem.mx/sites/default/files/inclusio n-y-atencion-diversidad/manual-azul.pdf

UAEM. (2012-2018b). Orientaciones Teóricas y Metodológicas esenciales para diseñar Adecuaciones Curriculares en Educación Superior. Programa Universitario en Educación Inclusiva y Atención a la Diversidad. Universidad Autónoma del Estado de Morelos: México.

http://www.uaem.mx/sites/default/files/orientac iones-teoricas.pdf 
Valle-Aparicio, J.E. (2014). Educación permanente: los programas universitarios para mayores en España como respuesta a una nueva realidad social. Revista de la Educación Superior. XLIII (3); No.171, 117-138. ISSN: 0185-2760.

https://doi.org/10.1016/j.resu.2015.03.003

Vázquez-Galicia, L. (2009). ¿Estudias y trabajas? Los estudiantes trabajadores de la Universidad Autónoma Metropolitana, Unidad Azcapotzalco. Revista Latinoamericana de Estudios Educativos (México).XXXIX (3-4), 121-149.

http://www.redalyc.org/pdf/270/27015078006.p df

Velis, L., Viteri, E., Terranova, E., \& Ordoñez, S. (2018). Nuevas tecnologías de la comunicación y capacidad cognitiva de adultos mayores. Universidad, Ciencia y Tecnología. 22

(89), 82-87. ISSN: 2542-3401. http://uctunexpo.autanabooks.com/index.php/uc t/article/view/35/38 